\title{
Compreensão de Emoções, Aceitação Social e Avaliação de Atributos Comportamentais em Crianças Escolares
}

\author{
Emotion Understanding, Social Acceptance and Evaluation \\ of Behavioral Attributes in School-Age Children
}

\author{
Gabriela Pavarini ${ }^{a}$, Carolina Piazzarollo Loureiro ${ }^{b}$ \& Débora de Hollanda Souza*,b \\ ${ }^{a}$ Universidade de Cambridge, Cambridge, Inglaterra \\ $\&{ }^{b}$ Universidade Federal de São Carlos, São Carlos, Brasil
}

\begin{abstract}
Resumo
Diversos pesquisadores do campo de estudos sobre teoria da mente têm investigado a relação entre compreensão de emoções e interação social. Seguindo essa linha de investigação, o presente estudo explorou a relação entre compreensão emocional, aceitação social e avaliação de atributos comportamentais pelos pares. Cinquenta e duas crianças escolares responderam a um teste de inteligência emocional e a dois testes de avaliação sociométrica. Não foi encontrada uma correlação entre a compreensão emocional e a aceitação social, porém uma correlação negativa entre a compreensão emocional e a avaliação de atributos ligados à agressividade/disruptividade foi observada. Esses resultados sugerem que o conhecimento de diferentes emoções e de estratégias de regulação emocional podem tornar as crianças menos propensas à agressividade reativa.

Palavras-chave: Compreensão de emoções; Aceitação social; Atributos comportamentais; Teoria da mente; Competência social.

Abstract

Several researchers in the theory of mind field have been investigating the relation between emotion understanding and social interaction. Following this line of investigation, the present study explored the relation between emotion understanding, social acceptance and evaluation of behavioral attributes by peers. Fifty-two school-aged children were administered an emotional intelligence test and two instruments of sociometric evaluation. A correlation between emotion understanding and social acceptance was not found, however, a negative correlation between emotion understanding and the evaluation of behavioral attributes related to aggressiveness/disruptivity was observed. The results_suggest that the knowledge of different emotions and of emotion regulation strategies may make children less prone to reactive aggressiveness.

Keywords: Emotion understanding; Social acceptance; Behavioral attributes; Theory of mind; Social competence.
\end{abstract}

As diferenças individuais no desenvolvimento de uma teoria da mente têm sido relacionadas a diversos aspectos do desenvolvimento social. A aquisição de uma teoria da mente relaciona-se positivamente à habilidade de brincar de forma cooperativa (Astington, 2003), a habilidades de interação social (Watson, Nixon, Wilson, \& Capage, 1999), a uma percepção positiva das experiências sociais na escola (Dunn, 1995), ao engajamento em brincadeiras de faz-de-conta (Astington \& Jenkins, 1999) e a variáveis familiares, como habilidades para lidar com conflitos com irmãos (Foote \& Holmes-Lonergan, 2003).

A compreensão dos estados mentais e respostas emocionais de outros indivíduos desempenha, portanto, um papel essencial na qualidade das relações interpessoais. Por outro lado, o desenvolvimento da teoria da mente parece ser influenciado justamente por essas relações interpessoais, mais especificamente, pelo engajamento das crianças em conversas envolvendo estados mentais (Brown, Donnelan-Maccall, \& Dunn, 1996; Dunn, 
Brown, Slomkowski, Tesla, \& Youngblade, 1991). Dessa forma, é plausível pensar que a participação em um sistema de pares pode promover ou facilitar a aquisição dessa habilidade cognitiva.

De fato, há algumas evidências de que a aquisição de uma teoria da mente esteja relacionada positivamente à aceitação em um grupo de amigos (e.g., Peterson \& Siegal, 2002) e essa associação parece ser bidirecional. Crianças mais atentas aos desejos, sentimentos e pensamentos de seus pares podem vir a ser mais bem avaliadas socialmente do que aquelas que não reconhecem esses estados mentais tão prontamente. Ao mesmo tempo, crianças mais populares e com relacionamentos mais positivos em um grupo de amigos podem ter mais oportunidades de interação e, portanto, aprimorar mais rapidamente sua compreensão de outras mentes. Já as crianças rejeitadas ou negligenciadas, por terem menos oportunidades de interações sociais saudáveis e, portanto, menos chance de trocar informações sobre estados mentais, podem apresentar uma compreensão social menos elaborada do que as crianças mais populares. Um atraso na compreensão de outras mentes pode, em decorrência, levar a dificuldades para fazer e manter amizades, levando a perpetuação de um status social de rejeitado ou de negligenciado.

A influência mútua entre teoria da mente e aceitação entre pares parece apresentar configurações distintas em cada período do desenvolvimento. Resultados de um estudo de Slaughter, Dennis e Pritchard (2002), por exemplo, revelaram uma relação entre teoria da mente (envolvendo compreensão de crenças, desejos e emoções) e popularidade apenas para as crianças com mais de 5 anos. Para os autores, esses resultados podem ser explicados em termos das mudanças desenvolvimentais no conceito de amizade. Enquanto para as crianças mais novas um amigo é aquele com quem se realiza atividades em comum, para as crianças mais velhas um amigo é alguém que fornece apoio e afeição (Furman \& Bierman, 1983) e com quem se compartilha pensamentos e sentimentos (Rose \& Asher, 2000). Assim, possivelmente a compreensão dos estados mentais das outras crianças torna-se cada vez mais relevante para estabelecer e manter relacionamentos próximos.

Estudos explorando a relação entre teoria da mente e aceitação entre pares no período escolar têm mostrado resultados consistentes. O sucesso em tarefas de compreensão de faux pas (insultos não-intencionais) por crianças de 8 e 9 anos, por exemplo, parece associar-se negativamente à rejeição de pares (Banerjee \& Watling, 2005). Nesse mesmo estudo, crianças rejeitadas ou com status controverso (avaliadas tanto positiva quanto negativamente) apresentaram desempenho significativamente inferior nessa tarefa em relação às outras crianças. Essa mesma relação não foi observada em crianças no período pré-escolar, especificamente com 5 e 6 anos.

Embora a literatura aponte, de modo geral, para um aumento da influência mútua entre a compreensão social e o relacionamento entre pares com a idade, ainda há controvérsia sobre quais componentes da teoria da mente são mais relevantes para essa relação. Badenes, Estevan e Bacete (2000), por exemplo, sugerem que crianças avaliadas como mais pró-sociais demonstram uma compreensão mais elaborada de mentiras brancas e de enganos (no inglês, deception). Por outro lado, o desempenho das mesmas em tarefas de crença falsa e ironia é semelhante ao das demais crianças nesta faixa etária. Em contraste, Diesendruck e Bem-Eliyahu (2006) observaram uma correlação entre uma avaliação positiva pelos pares e a compreensão de crença falsa, mas não uma associação com a compreensão de motivos subjacentes às ações ou com a tomada de perspectiva afetiva. Ademais, enquanto o primeiro estudo indicou uma correlação negativa entre compreensão de mentiras brancas (porém não de crença falsa ou de ironia) e uma avaliação negativa pelos pares, o segundo indicou correlação negativa entre agressividade/disruptividade e a compreensão de emoções positivas.

Assim, algumas habilidades específicas ligadas à cognição social, como a compreensão de intencionalidade e de mentiras brancas, podem ser preditoras mais diretas do modo como as crianças se relacionam com seus pares. Outra habilidade que pode ser especialmente importante neste sentido e que foi pouco explorada durante o período escolar é a compreensão de emoções.

O tema da compreensão de emoções é de notável interesse nos estudos sobre teoria da mente e comportamento social, já que "compreender estados cognitivos, como perceber que alguém é ignorante ou está enganado, é insuficiente para determinar como se comportar diante da pessoa. É preciso compreender também como eles se sentem em relação à situação" (Astington, 2003, p. 34). Essa compreensão parece ser fundamental para a qualidade das relações interpessoais estabelecidas entre crianças em um grupo de amigos. Da mesma forma, a aceitação dos pares aumenta a oportunidade das crianças aprenderem sobre emoções (J. Cassidy, Parke, Butkovsky, \& Braungart, 1992).

A compreensão de emoções pode ser definida como "a habilidade sociocognitiva de reconhecer e interpretar as emoções dos outros" (Martin \& Green, 2005, p. 229). No presente estudo, ela é entendida como um conceito amplo, que reúne uma série de habilidades e compreensões relacionadas à emoção, como a identificação de emoções, compreensão de causas externas das emoções, compreensão da relação entre emoções e outros estados mentais (e.g., desejos, crenças), conhecimento de estratégias de regulação das emoções e compreensão de respostas emocionais ambivalentes (Pons, Harris, \& Rosnay, 2004). Como pode ser observado, essa perspectiva nos remete inevitavelmente à necessidade de discutir as interfaces entre cognição e emoção.

A visão tradicional é a de que não há uma associação inerente entre cognição e emoção, ou seja, são domínios 
independentes (LeDoux, 1996). Evidências mais recentes, no entanto, sugerem que até mesmo discriminações afetivas básicas requerem input cognitivo. Com base nesses resultados, uma nova proposta, a de que esses dois processos são complementares, tem se fortalecido (Adolphs \& Damasio, 2001; Storbeck \& Clore, 2007). Nesse contexto, o tema da compreensão de emoções adquire especial relevância ao investigar processos que são necessariamente cognitivos e emocionais.

A compreensão de emoções parece ter um papel importante em ajudar as crianças a se relacionarem com seus pares de forma mais sensível. Essa sensibilidade pode ser interpretada em termos de cognição social, isto é, as crianças diferem em sua compreensão de emoções-chave e este conhecimento as ajuda a lidar com os outros (Harris, 2000). De fato, a capacidade de identificar reações emocionais típicas e atípicas a diversas situações, um componente importante da compreensão de emoções, mostrou-se um preditor direto da popularidade de crianças pré-escolares (Denham, MacKinley, Couchoud, \& Holt, 1990).

Conforme apresentado anteriormente, é possível prever que essa relação seja ainda mais evidente em crianças de idade escolar. O início da escolaridade, em especial, parece ser um período importante para uma mudança no conceito de amizade e no tipo de relação interpessoal estabelecida entre pares (Furman \& Bierman, 1983). O presente estudo pretende contribuir nessa direção investigando, em uma amostra de crianças escolares, a relação entre dois domínios da compreensão de emoções (experiencial e estratégico), a aceitação social e a forma como as crianças são avaliadas pelos pares em termos de atributos comportamentais relevantes à interação social.

\section{Método}

\section{Participantes}

Participaram do presente estudo 52 crianças (25 meninas e 27 meninos) que frequentavam uma escola de Ensino Fundamental da rede particular de um município do estado de São Paulo. As crianças foram divididas em três grupos, de acordo com a série: 21 da $3^{\text {a }}$ série $(M=8,9$ anos; $D P=0,34), 17$ da $4^{\text {a }}$ série $(M=9,9$ anos; $D P=$ $0,35)$ e 14 da $5^{\text {a }}$ série $(M=10,9$ anos; $D P=0,33)$. A pesquisa foi aprovada pelo Comitê de Ética em Pesquisa com Seres Humanos e participaram do estudo apenas as crianças que apresentaram o consentimento por escrito dos pais.

\section{Compreensão de Emoções}

Para medir a compreensão de emoções foi utilizado o Teste de Inteligência Emocional para Crianças (TIEC), criado e validado para a população brasileira por Bueno (2008). O teste mede dois componentes gerais da inteligência emocional infantil: o experiencial e o estratégico. O primeiro abrange itens relativos à percepção de emo- ções (a capacidade de identificar uma emoção) e à utilização da emoção para facilitação do pensamento (que inclui a capacidade de associar emoções a sensações térmicas, gustativas, etc., e a eventos do cotidiano). No geral, esses itens referem-se a emoções negativas.

O componente estratégico abrange itens relacionados ao conhecimento sobre diferentes emoções e a associação dessas com eventos desencadeadores, além do conhecimento de estratégias de regulação emocional (capacidade de identificar estratégias de coping apropriadas para resolução de um problema). Os itens desse fator envolvem mais de uma emoção ou misturas de emoções e, no geral, referem-se a emoções positivas.

O teste completo é composto por tarefas de desempenho que se referem a oito histórias que retratam as emoções de alegria, tristeza, raiva, medo, ansiedade, aceitação, aversão e surpresa. As histórias são apresentadas às crianças no decorrer do procedimento, utilizando-se um áudio previamente gravado e a apresentação de figuras ilustrativas. No presente estudo, o instrumento foi aplicado com o auxílio de um computador para facilitar a apresentação das figuras às crianças.

Antes de iniciar a primeira história, a experimentadora distribuía uma folha de respostas para a criança e explicava a tarefa. Mostrava a ela algumas figuras na tela e a história em áudio. Em seguida, lia a questão e as alternativas apresentadas na tela e aguardava até que a criança marcasse a sua resposta na folha previamente distribuída. Repetia-se o procedimento para as demais questões e todo o processo para cada uma das histórias.

Cada item do TIEC foi pontuado dicotomicamente (zero pontos para erro, um para acerto) e através da soma dos itens correspondentes a cada fator calculou-se o desempenho das crianças nos componentes experiencial e estratégico da inteligência emocional. $O$ teste foi aplicado no presente estudo quando o instrumento ainda estava em fase de validação, compreendendo 58 itens. Destes, apenas 38 foram utilizados para o cálculo da pontuação das crianças, sendo 19 para o componente experiencial e a mesma quantidade para o estratégico (ver Bueno \& Primi, 2009, para uma análise das propriedades psicométricas do instrumento).

\section{Avaliação pelos Pares}

Avaliação Sociométrica. O status sociométrico de cada criança foi atribuído conforme o método de Avaliação Sociométrica de Coie e Dodge (1983). Para a realização do procedimento, todas as crianças participantes do estudo foram fotografadas individualmente e as fotografias eram apresentadas na tela de um computador. A criança entrevistada era solicitada a nomear cada uma das crianças participantes que faziam parte de sua sala de aula, através das fotografias. Em seguida, ela era solicitada a nomear as três crianças com as quais ela mais gostava de brincar junto e três com as quais ela não gostava muito de brincar. 
Cada nomeação, positiva (mais gosta) ou negativa (menos gosta), recebida equivaleu a um ponto. Para cada criança, então, foi calculado um escore bruto da quantidade de nomeações positivas e outro referente ao número de nomeações negativas recebidas, isto é, da quantidade de vezes que o nome daquela criança havia sido mencionado pelos colegas em cada categoria. Como o número de crianças para cada grupo de idade era diferente, esses escores foram normatizados em um intervalo idêntico para os três grupos.

Para o cálculo da preferência social (grau em que as crianças são "lembradas positivamente" pelos pares), subtraiu-se a quantidade de nomeações negativas do número de nomeações positivas recebidas. O impacto social (grau em que as crianças são "lembradas" pelos colegas, independente de serem avaliadas de forma positiva ou negativa) foi calculado através da soma das nomeações positivas e negativas.

Os valores padronizados (escores $z$ ) das nomeações positivas (NP) e negativas (NN), da preferência social (PS) e do impacto social (IS) foram também calculados, para a atribuição do status sociométrico de cada participante. As crianças foram alocadas em cinco grupos, de acordo com o critério sugerido por Coie e Dodge (1983): (a) popular: crianças com escore de $\mathrm{PS}>1$; NP $>0$ e NN $<0$; (b) rejeitado: crianças com escore de $\mathrm{PS}<-1$; NP $<0$ e NN $>0$; (c) negligenciado: crianças com escores de IS $<-1 ; \mathrm{NP}<0$ e $\mathrm{NN}<0$; (d) controverso: crianças com escores de IS $>1$; $\mathrm{NP}>0$ e NN $>0$; e (e) intermediário: as demais crianças, que não atenderam aos requisitos dos outros grupos.

Avaliação da Competência Social. Para medir a forma como as crianças eram avaliadas pelos pares em relação a características comportamentais relevantes à interação social, utilizou-se o Revised Class Play (RCP). O instrumento RCP, desenvolvido por Masten, Morrison e Pellegrini (1985) e traduzido e adaptado para o Brasil por Pizzinato (2003), avalia, de forma sociométrica, três dimensões da competência social infantil entre pares: popularidade/liderança, agressividade/disruptividade e sensibilidade/isolamento.

$\mathrm{O}$ teste foi aplicado por intermédio de um computador, sendo as fotos das crianças de cada grupo de amigos apresentadas na tela, juntamente com as perguntas. A experimentadora fazia 34 perguntas de caráter nominal, em que a criança era solicitada a indicar o nome do amigo que mais se identificasse com a característica apresentada na questão. Quinze questões referiam-se à categoria popularidade/liderança (e.g., "quem é bom líder?", "quem tem muitos amigos?"), nove indicavam características relativas à agressividade/disruptividade (e.g., "quem bate em outras crianças?", "quem mete o nariz onde não é chamado?") e as dez questões restantes eram referentes à sensibilidade/isolamento (e.g., “quem é muito tímido?", “quem se magoa facilmente?”).
Cada uma das nomeações recebidas pelo participante equivaleu a um ponto. Através da somatória das nomeações recebidas nos itens referentes a cada categoria do teste, obteve-se os escores de cada participante para popularidade/liderança, agressividade/disruptividade e sensibilidade/isolamento. Os escores foram normatizados em um mesmo intervalo para os três grupos de idade e as três categorias do teste.

\section{Resultados}

$\mathrm{O}$ desempenho dos participantes nas tarefas referentes ao componente experiencial $(M=14,63, D P=2,27)$ da inteligência emocional foi significativamente superior ao desempenho nas tarefas relativas ao componente estratégico $(M=13,48, D P=3,22), t(51)=-2,23 ; p<0,05$. Análises multivariadas revelaram um efeito de idade para a o componente experiencial da inteligência emocional $(F(2,49)=6,01, p<0,01)$ e comparações emparelhadas mostraram que o escore do grupo de 9 anos $(M=11,76$; $D P=0,64)$ foi inferior ao do grupo de 10 anos $(M=14,59$; $D P=0,71 ; p<0,05)$ e de 11 anos $(M=14,71 ; D P=0,79$; $p<0,05)$. Não se observou efeito de idade para o componente estratégico $(F(2,49)=0,31, p=$ n.s. $)$.

Em relação à Avaliação Sociométrica, observou-se uma quantidade variável de crianças nas cinco categorias sociométricas $(\chi 2(4)=21,13 ; p<0,001)$. A maioria das crianças foi alocada nas categorias intermediário e popular e observou-se um número inferior de crianças nas categorias negligenciado e controverso. A Tabela 1 apresenta uma comparação entre as porcentagens das crianças deste estudo nas cinco categorias sociométricas, incluindo também as de um grupo de crianças americanas com idade correspondente (Coie \& Dodge, 1983). A distribuição sociométrica observada no grupo de crianças brasileiras foi estatisticamente semelhante à observada no grupo americano $\left(\chi^{2}(4)=4,17 ; p=\right.$ n.s. $)$.

\section{Tabela 1}

Comparação entre a Frequência Relativa de Crianças Brasileiras e Americanas em cada Status Sociométrico

\begin{tabular}{lcc}
\hline Status sociométrico & $\begin{array}{c}\text { Crianças } \\
\text { brasileiras (\%) }\end{array}$ & $\begin{array}{c}\text { Crianças } \\
\text { americanas (\%) }\end{array}$ \\
\hline Popular & 26,9 & 23,0 \\
Intermediário & 32,7 & 30,0 \\
Rejeitado & 21,2 & 21,0 \\
Negligenciado & 11,5 & 19,5 \\
Controverso & 7,7 & 6,5 \\
\hline
\end{tabular}

Quanto às escolhas sociométricas, tanto as meninas quanto os meninos nomearam mais crianças do mesmo sexo do que do sexo oposto como aquelas com quem mais 
gostavam de brincar $(t(24)=18,94 ; p<0,001$ para as meninas, e $t(27)=17,71 ; p<0,001$ para os meninos). No caso das escolhas sociométricas negativas, porém, uma diferença de gênero foi observada. As meninas nomearam mais crianças do sexo oposto do que do mesmo sexo como aquelas com quem não gostavam muito de brincar $(t(23)=-4,92 ; p<0,001)$, enquanto para os meninos, nenhuma diferença significativa foi observada $(t(27)=$ 1,$09 ; p=$ n.s.).

Uma relação significativa entre a avaliação sociométrica das crianças e as variáveis medidas através do Revised Class Play foi encontrada (ver Tabela 2). Houve uma correlação positiva entre a preferência social das crianças e os escores de popularidade/liderança das mesmas. Adicionalmente, obteve-se uma correlação negativa significativa entre preferência social e agressividade/disruptividade. O impacto social, por sua vez, apresentou uma correlação positiva com o escore de agressividade/disruptividade. O escore total do RCP também apresentou uma correlação positiva com a categoria impacto social $(r=0,44 ; p<0,001)$. Observouse, ainda, que quanto maior o escore de popularidade/ liderança, maior o número de nomeações positivas recebidas, além de haver uma tendência a um menor número de nomeações negativas. Do mesmo modo, observou-se uma correlação positiva entre a quantidade de nomeações negativas e a agressividade/disruptividade dos participantes.

Tabela 2

Correlações (r) entre as Categorias do Revised Class Play e a Avaliação Sociométrica

\begin{tabular}{lcccc}
\hline Revised Class Play & $\begin{array}{c}\text { Preferência } \\
\text { social }\end{array}$ & $\begin{array}{c}\text { Impacto } \\
\text { social }\end{array}$ & $\begin{array}{c}\text { Nomeações } \\
\text { positivas }\end{array}$ & $\begin{array}{c}\text { Nomeações } \\
\text { negativas }\end{array}$ \\
\hline Popularidade/liderança & $0,41^{* *}$ & 0,04 & $0,48^{* * *}$ & $-0,27^{+}$ \\
Agressividade/disruptividade & $-0,49^{* * *}$ & $0,42^{* *}$ & $-0,21$ & $0,54^{* * *}$ \\
Sensibilidade/isolamento & $-0,11$ & 0,18 & 0,02 & 0,16 \\
\hline
\end{tabular}

Nota. ${ }^{* * *} p<0,001 ;{ }^{* *} p<0,005 ;{ }^{+} p=0,056$.

Inteligência Emocional, Aceitação Social

e Avaliação de Atributos Comportamentais

Contrariamente ao esperado, as análises não indicaram uma correlação significativa entre o desempenho das crianças no domínio experiencial da inteligência emocional e a aceitação pelos pares, tanto em relação à preferência social $(r=-0,05 ; p=$ n.s. $)$ quanto ao impacto social $(r=0,19 ; p=$ n.s. $)$. O mesmo foi observado para a correlação entre o domínio estratégico da inteligência emocional e a aceitação de pares $(r=0,05$ para preferência social e $r=0,01$ para impacto social, $p s=$ n.s.). De modo semelhante, não se observou um efeito do status sociométrico sobre os escores do componente experiencial $(F(4,51)=0,29 ; p=$ n.s. $)$ e estratégico $(F(4,51)=$ 0,$98 ; p=$ n.s.) da inteligência emocional.

As análises realizadas não revelaram uma relação significativa entre o desempenho dos participantes no componente experiencial do TIEC e os escores obtidos nas três categorias do Revised Class Play $(r=0,18$ para popularidade/liderança, $r=0,11$ para agressividade/ disruptividade e $r=0,15$ para sensibilidade/isolamento, $p s=$ n.s). O desempenho no domínio estratégico do TIEC não se correlacionou de forma significativa com os escores na categoria popularidade/liderança $(r=0,09 ; p=$ n.s.), nem tampouco de sensibilidade/isolamento $(r=$ - 0,$15 ; p=$ n.s.). Identificou-se, porém, uma correlação significativa entre o desempenho dos participantes no domínio estratégico e a agressividade/disruptividade $(r=-0,36, p<0,01)$.

\section{Discussão}

\section{Inteligência Emocional}

Comparando os escores nas duas categorias do TIEC, verifica-se que as crianças obtiveram um desempenho superior nos itens referentes ao componente experiencial do que nos itens referentes ao componente estratégico. O componente experiencial envolvia especialmente a percepção de emoções e a capacidade de relacioná-las com aspectos ambientais, habilidades mais primárias da compreensão de emoções. Já as tarefas do componente estratégico exigiam a compreensão de sutilezas que distinguem uma emoção da outra, uma habilidade mais sofisticada que, de fato, emerge mais tarde no desenvolvimento infantil (Pons et al., 2004).

Observou-se, ainda, que as crianças de 10 e 11 anos tiveram um desempenho significativamente superior ao das crianças mais novas no domínio experiencial. Essa mesma diferença de desempenho já havia sido observada por Bueno e Primi (2009) indicando que esse período é especialmente relevante para a aquisição de habilidades relacionadas à percepção de emoções e à associação dessas com aspectos ambientais.

\section{Aceitação Social e Avaliação de Atributos Comportamentais}

Os resultados indicaram algumas evidências de validade de construto dos instrumentos utilizados para avaliação pelos pares. As evidências de validade convergente 
incluíram correlações significativas entre as categorias da Avaliação Sociométrica de Coie e Dodge (1983) e as categorias do Revised Class Play, em especial: (a) a correlação positiva entre as categorias preferência social e popularidade/liderança; (b) a correlação negativa entre preferência social e agressividade/disruptividade; e (c) a correlação positiva entre o escore total no RCP e o impacto social. Esses resultados são particularmente importantes uma vez que o RCP é um instrumento ainda não validado no Brasil.

É interessante apontar que a frequência relativa de crianças localizadas em cada status sociométrico foi similar à encontrada em um estudo com crianças americanas de idade correspondente (Coie \& Dodge, 1983). Estudos futuros com amostras transculturais poderiam explorar de forma mais aprofundada semelhanças e diferenças na forma como as crianças são avaliadas por seus pares. Porém, algumas semelhanças entre características da avaliação sociométrica de crianças brasileiras e americanas já foram observadas em outros estudos, como a preferência por colegas de mesmo sexo e nomeação negativa de mais colegas do sexo oposto (Guzman, Carlo, Ontai, Koller, \& Knight, 2004).

Esses resultados foram igualmente observados no presente estudo, porém com uma diferença de gênero para as nomeações negativas. As crianças do sexo feminino nomearam negativamente mais colegas do sexo oposto, enquanto para os meninos não se observou um efeito de gênero. Estudos futuros devem explorar as variáveis que contribuem para essas diferenças nas preferências por amigos entre as crianças brasileiras.

A análise dos resultados demonstrou também que a categoria popularidade/liderança do teste RCP estava positivamente correlacionada à quantidade de nomeações positivas e negativamente correlacionada à quantidade de nomeações negativas recebidas. Da mesma forma, a categoria agressividade/disruptividade estava correlacionada positivamente com a quantidade de nomeações negativas. Esses resultados corroboram os de Morais, Otta e Scala (2001) que apontam para correlações entre avaliação sociométrica e a avaliação de atributos comportamentais. Mais especificamente, foram encontradas correlações entre avaliações positivas e atributos comportamentais positivos como "tem muitos amigos" ou "lidera outras crianças" (igualmente presentes na categoria popularidade/liderança do RCP), bem como entre avaliações negativas e atributos comportamentais negativos, tais como "provoca brigas" e "tem o 'pavio' curto". Esses últimos são semelhantes a alguns itens da categoria agressividade/disruptividade do RCP (e.g., "se envolve em muitas brigas", "provoca muito outras crianças" e "perde a paciência facilmente").

$\mathrm{Na}$ mesma direção, outros estudos têm indicado que crianças que apresentam mais comportamentos pró-sociais são preferidas pelos pares e, em contraste, aquelas que apresentam mais comportamentos anti-sociais so- frem maior rejeição pelos colegas (Alderfer, Wiebe, \& Hartmann, 2001; Pakaslahti, Karjalainen, \& KeltikangasJärvinen, 2002). Há, ainda, claras evidências de que a competência social está positivamente associada à aceitação entre pares e a relacionamentos interpessoais saudáveis (ver Hubbard \& Coie, 1994, para uma revisão). Assim, o desempenho social das crianças em situações de interação com os pares parece ser um preditor confiável da aceitação social.

Os resultados do presente estudo sugeriram ainda que quanto mais agressivas/disruptivas as crianças eram avaliadas pelos colegas, maior o seu impacto social. $\mathrm{O}$ impacto social é calculado somando-se as nomeações positivas e negativas recebidas pelos participantes. É plausível supor que a grande quantidade de avaliações negativas recebidas por essas crianças tenha sido o fator determinante na relação entre agressividade/disruptividade e impacto social. Ressalta-se, no entanto, que não se observou uma relação negativa entre a quantidade de nomeações positivas e a agressividade/disruptividade. Levanta-se a hipótese de que estas crianças percebidas como agressivas/disruptivas e com elevado impacto social agem de maneira pró-social apenas dentro de seu grupo específico de colegas e de maneira agressiva com o restante deles, um padrão anteriormente descrito por Repacholi, Slaughter, Pritchard e Gibbs (2003). Assim, além de serem "lembradas" negativamente pelos colegas, elas receberiam nomeações positivas por parte de seu grupo mais restrito de amigos.

\section{Inteligência Emocional, Aceitação Social e Atributos Comportamentais}

Contrariamente ao esperado, as análises não indicaram uma correlação entre o desempenho dos participantes no TIEC e os escores de aceitação social, popularidade/liderança ou sensibilidade/isolamento. Esperava-se que as crianças com maior grau de popularidade ou avaliadas mais positivamente apresentassem uma compreensão de emoções mais elaborada do que as crianças com status intermediário ou rejeitado, já que há algumas evidências na literatura de uma relação entre diferentes componentes da teoria da mente e a popularidade (e.g., Edwards, Manstead, \& MacDonald, 1984).

Resultados semelhantes aos observados no presente estudo, porém, já foram observados por outros autores (e.g., Badenes et al., 2000; Diesendruck \& Bem-Eliyahu, 2006), embora os dados sobre esse tema sejam pouco consensuais. Um estudo com pré-adolescentes, por exemplo, não revelou uma relação significativa entre teoria da mente e popularidade controlando-se o efeito da competência verbal (Bosacki \& Astington, 1999). Os participantes que apresentavam uma compreensão social mais elaborada, por outro lado, foram avaliados pelos pares como mais hábeis em lidar com situações sociais.

Um estudo semelhante não indicou uma relação significativa entre a freqüência de comportamentos de coo- 
peração, ajuda e partilha e a compreensão de emoções, bem como entre esta variável e a popularidade (K. Cassidy, Werner, Rourke, Zubernis, \& Balaraman, 2003). Curiosamente, as crianças com uma compreensão de emoções mais elaborada eram mais propensas a serem alvos de comportamentos pró-sociais emitidos por seus pares. Esses resultados indicam que, embora seja possível que a compreensão de emoções não necessariamente leve a uma maior popularidade ou à adoção de comportamentos pró-sociais, ela pode ser uma habilidade importante para conseguir ajuda, apoio e cooperação dos colegas.

Possivelmente, a capacidade de identificar e prever as emoções dos colegas permite que as crianças desenvolvam estratégias mais efetivas para regular suas interações sociais, o que, por sua vez, pode incentivar esses mesmos colegas a emitirem comportamentos prósociais direcionados a elas (e.g., ajudar em uma tarefa escolar difícil). De acordo com essa hipótese, há evidências de que as crianças com uma compreensão sofisticada de estados mentais são mais propensas a apresentar estratégias mais elaboradas de interação social e resolução de conflitos, além de apresentarem uma comunicação verbal mais bem-sucedida na interação com os colegas (cf. Dunn, 1994).

Dessa forma, torna-se compreensível que crianças mais hábeis em compreender estados mentais, por apresentarem estratégias mais elaboradas de interação social, ajam de maneira menos agressiva em relação aos pares. No presente estudo, uma correlação negativa entre o desempenho das crianças no componente estratégico da inteligência emocional e o índice de agressividade/disruptividade foi encontrada. O componente estratégico incluía tanto o conhecimento sobre diferentes emoções e associação com eventos desencadeadores, quanto o conhecimento de estratégias de regulação emocional. Esta última habilidade pode favorecer o controle de emoções negativas pelas crianças em situações de conflito com pares, evitando interações agressivas. Pesquisas anteriores já haviam demonstrado uma relação negativa entre a utilização de estratégias de regulação de emoções e a freqüência de comportamentos agressivos (Calkins, Gill, Johnson, \& Smith, 1999).

Quanto à relação entre o conhecimento sobre diferentes emoções e a agressividade/disruptividade, no entanto, os resultados da literatura apresentam alguma inconsistência. Autores como Denham et al. (2002), por exemplo, observaram que déficits na compreensão de situações emocionais estão relacionados a uma maior freqüência de comportamentos agressivos durante o período pré-escolar. Por outro lado, outras pesquisas não indicaram uma relação consistente entre a compreensão da mente e a agressividade (Monks, Smith, \& Swettenham, 2005) ou, ainda, indicaram uma relação positiva entre essas variáveis (Sutton, Smith, \& Swettenham, 1999b). Tal incongruência possivelmente está relacionada ao tipo de comportamento agressivo sendo avaliado (cf. Blair, 2004).

A maioria dos itens do instrumento Revised Class Play avaliava atributos comportamentais relacionados à agressividade/disruptividade reativa (e.g., "bate em outras crianças", "sentimentos explodem facilmente", "perde a paciência facilmente"). A agressividade reativa diz respeito a reações anti-sociais impulsivas e defensivas, em resposta a frustrações ou ameaças, e se diferencia da agressividade instrumental, associada a comportamentos agressivos deliberados, direcionados para uma finalidade (Crick \& Dodge, 1996). É plausível supor que uma compreensão mais elaborada de emoções e de estratégias de regulação emocional seja um fator protetivo para a agressividade reativa.

Essa relação entre compreensão de emoções e agressividade reativa pode, também, ser mediada por um viés de atribuição. Há evidências de que dificuldades na compreensão de emoções tornam as crianças mais propensas a interpretarem comportamentos ambíguos de seus pares como propositais e hostis (Perkins, 2003). Crianças que apresentam um viés de atribuição hostil normalmente reagem de maneira mais agressiva em relação aos seus pares e esse viés de atribuição é mais frequente em crianças que apresentam comportamentos agressivos reativos do que nas que apresentam comportamentos agressivos instrumentais (Crick \& Dodge, 1996; Hubbard, Dodge, Cillessen, Coie, \& Schwartz, 2001). Assim, uma compreensão de emoções mais elaborada e um viés atribucional adequado podem proteger as crianças de apresentarem explosões de raiva e agressividade em resposta a frustrações ou ameaças.

Em contraste, há evidências de que uma compreensão de emoções sofisticada não é efetiva para inibir a agressividade instrumental. Crianças que praticam bullying, por exemplo, podem apresentar intacta a capacidade de compreender outras mentes e, simultaneamente, um desempenho social inadequado. Uma teoria da mente sofisticada pode até mesmo estar subjacente a muitos atos agressivos instrumentais: "o contexto e as habilidades do bullying são em grande parte baseados na habilidade de compreender e manipular as mentes dos outros - uma 'teoria da mente' ou cognição social" (Sutton, Smith, \& Swettenham, 1999a, p. 120).

A compreensão de outras mentes pode, assim, tanto ser preditora de comportamentos socialmente adequados quanto de ações moralmente repreensíveis. Ronald, Happé, Hughes e Plomin (2005), através de uma análise fatorial de comportamentos pró-sociais e anti-sociais, identificaram três fatores, definidos como: (a) comportamentos pró-sociais que exigem uma teoria da mente (e.g., "Considera os sentimentos das outras pessoas"); (b) comportamentos anti-sociais que exigem uma teoria da mente (e.g., "Culpa os outros pelas coisas"); e (c) comportamentos pró e anti-sociais que não exigem uma teoria da mente (e.g., "É gentil com os animais", "Destrói as 
coisas dos outros"). Essa análise clarifica resultados controversos da literatura sobre teoria da mente e aceitação social, tornando plausível afirmar que a compreensão de emoções por si só não seja suficiente para prever um desempenho social adequado e, em conseqüência, uma maior aceitação entre os colegas.

É importante destacar, no entanto, que o presente estudo foi realizado com crianças de classe média, frequentadoras de uma escola particular no estado de São Paulo. Futuras pesquisas devem ser conduzidas com crianças de diferentes níveis socioeconômicos ou em diferentes contextos culturais para se averiguar se os resultados encontrados podem ser, de fato, generalizados. É possível, por exemplo, que as atribuições comportamentais avaliadas pelo Revised Class Play (e.g., "quem mete o nariz onde não é chamado?" ou “quem se magoa facilmente?") sejam influenciadas pelo contexto sociocultural dessas crianças.

Concluindo, o presente estudo indica que a compreensão de emoções não está diretamente relacionada à aceitação social ou a uma avaliação mais positiva pelos colegas. Por outro lado, sugere que atributos comportamentais positivos (e.g., "tem muitos amigos", "alguém em quem se pode confiar") e atributos anti-sociais (e.g., "briga com os outros"; "bate em outras crianças") podem ser preditores confiáveis da aceitação e rejeição social, respectivamente. $O$ presente estudo sugere, ainda, que o conhecimento de estratégias de regulação emocional, como avaliado pelo componente estratégico do TIEC, pode tornar as crianças menos propensas à agressividade reativa, um efeito possivelmente mediado por estratégias mais elaboradas de interação social e de resolução de conflitos e um viés atribucional adequado.

\section{Referências}

Adolphs, R., \& Damasio, A. R. (2001). The interacton of affect and cognition: A new biological perspective. In J. P. Forgas (Ed.), Handbook of affect and social cognition (pp. 27-49). Mahwah, NJ: Lawrence Erlbaum.

Alderfer, M., Wiebe, D. J., \& Hartmann, D. P. (2001). Social behaviour and illness information interact to influence the peer acceptance of children with chronic illness. Bristish Journal of Health Psychology, 6, 243-255.

Astington, J.W. (2003). Sometimes necessary, never sufficient: False-belief understanding and social competence. In B. Repacholi \& V. Slaughter (Eds.), Individual differences in theory of mind (pp. 13-38). New York: Psychology Press.

Astington, J. W., \& Jenkins, J. M. (1999). A longitudinal study of the relation between language and theory-of-mind development. Developmental Psychology, 35, 1311-1320.

Badenes, L. V., Estevan, R. A. C., \& Bacete, F. J. G. (2000). Theory of mind and peer rejection at school. Social Development, 8, 271-283.

Banerjee, R., \& Watling, D. (2005). Children's understanding of faux pas: Associations with peer relations. Hellenic Journal of Psychology, 2(1), 27-45.
Blair, R. J. R. (2004). Did cain fail to represent the thoughts of Abel before he killed him? The relationship between theory of mind and aggression. In B. Repacholi \& V. Slaughter (Eds.), Individual differences in theory of mind (pp. 143-170). New York: Psychology Press.

Bosacki, S. B., \& Astington, J. W. (1999). Theory of mind in preadolescence: Relations between social understanding and social competence. Social Development, 8, 237-253.

Brown, J. R., Donelan-Maccal, N., \& Dunn, J. (1996). Why talk about mental states? The significance of children conversation with friends, siblings and mothers. Child Development, 67, 836-849.

Bueno, J. M. H. (2008). Construção de um instrumento para avaliação da inteligência emocional em crianças. Tese de Doutorado não-publicada, Universidade São Francisco, Itatiba, SP.

Bueno, J. M. H., \& Primi, R. (2009). Analysis of the psychometric properties of an Emotional Intelligence Test for Children (EITC). Manuscript submitted for publication.

Calkins, S. D., Gill, K. L., Johnson, M. C., \& Smith, C. L. (1999). Emotional reactivity and emotion regulation strategies as predictors of social behaviors with peers during toddlerhood. Social Development, 8, 310-341.

Cassidy, J., Parke, R. D., Butkovsky, L., \& Braungart, J. M. (1992). Family-peer connections: The roles of emotional expressiveness within the family and children's understanding of emotions. Child Development, 63, 603-618.

Cassidy, K., Werner, R., Rourke, M., Zubernis, L., \& Balaraman, G. (2003). The relationship between psychological understanding and positive social behavior. Social Development, 12, 198-221.

Coie, J. D., \& Dodge, K. A. (1983). Continuities and changes in children's social status. Merril-Palmer Quarterly, 29, 261-282.

Crick, N. R., \& Dodge, K. A. (1996). Social information processing mechanisms in reactive and proactive aggression. Child Development, 67, 993-1002.

Denham, S. A., Caverly, S., Schmidt, M., Blair, K., DeMulder, E., Caal, S., et al. (2002). Preschool understanding of emotions: Contributions to classroom anger and aggression. Journal of Child Psychology and Psychiatry, 43, 901-916.

Denham, S. A., McKinley, M., Couchoud, E. A., \& Holt, R. (1990). Emotional and behavioral predictors of preschool peer ratings. Child Development, 61, 1145-1152.

Diesendruck, G., \& Ben-Eliyahu, A. (2006). The relationships among social cognition, peer acceptance, and social behavior in Israeli kindergartners. International Journal of Behavioral Development, 30, 137-147.

Dunn, J. (1995) Children as psychologists: The later correlates of individual differences in understanding of emotions and other minds. Cognition and Emotion, 9, 187-201.

Dunn, J. (2004). Children's friendships: The beginnings of intimacy. Malden, MA: Blackwell.

Dunn, J., Brown, J. R., Slomkowski, C., Tesla, C., \& Youngblade, L. (1991). Young children's understanding of other people's feelings and beliefs: Individual differences and their antecedents. Child Development, 62, 1352-1366.

Edwards, R., Manstead, A. S. R., \& MacDonald, C. J. (1984). The relationship between children's sociometric status and ability to recognize facial expressions of emotion. European Journal of Social Psychology, 14, 235-238.

Foote, R. C., \& Holmes-Lonergan, H. A. (2003). Sibling conflict and theory of mind. British Journal of Developmental Psychology, 21, 45-58. 
Furman, W., \& Bierman, K. L. (1983). Developmental changes in Young Children's conceptions of friendship. Child Development, 54, 549-556.

Guzman, M. R. T., Carlo, G., Ontai, L. L., Koller, S. H., \& Knight, G. P. (2004). Gender and age differences in Brazilian children's friendship nominations and peer sociometric ratings. Sex Roles, 51, 217-225.

Harris, P. L. (2000). Understanding emotion. In M. Lewis \& J. Haviland-Jones (Eds.), Handbook of emotions (pp. 281-292). New York: The Guilford Press.

Hubbard, J. A., \& Coie, J. D. (1994). Emotional correlates of social competence in children's peer relationships. MerrillPalmer Quarterly, 40, 1-20.

Hubbard, J. A., Dodge, K. A., Cillessen, A. H., Coie, J. D., \& Schwartz, D. (2001). The dyadic nature of social information processing in boys' reactive and proactive aggression. Journal of Personality and Social Psychology, 80, 268-280.

LeDoux, J. (1996). The emotional brain: The mysterious underpinnings of emotional life. New York: Simon \& Schuster.

Martin, R. M., \& Green, J. A. (2005). The use of emotion explanations by mothers: Relation to preschoolers' gender and understanding of emotions. Social Development, 14, 229249.

Masten, A. S., Morrison, P., \& Pellegrini, D. S. (1985). A Revised Class Play Method of peer assessment. Developmental Psychology, 21, 523-533.

Monks, C. P., Smith, P. K., \& Swettenham, J. (2005). The psychological correlates of peer victimisation in preschool: Social cognitive skills, executive function and attachment profiles. Aggressive Behavior, 31, 571-588.

Morais, M. L. S., Otta, E., \& Scala, C. T. (2001). Status sociométrico e descrições comportamentais por pares: Um estudo sobre a competência social de pré-escolares. Psicologia: Reflexão e Crítica, 14, 119-131.

Pakaslahti, L., Karjalainen, A., \& Keltikangas-Järvinen, L. (2002). Relationships between adolescent prosocial problem-solving strategies, prosocial behaviour, and social acceptance. International Journal of Behavioral Development, 26, 137-144.

Perkins, D. M. (2003). Effect of parenting, emotional intelligence, hostile attributional bias, and peer status on problem behavior. Unpublished doctoral dissertation, University of New Hampshire, Manchester, NH.

Peterson, C. C., \& Siegal, M. (2002). Mindreading and moral awareness in popular and rejected preschoolers. British Journal of Developmental Psychology, 20, 205-224.

Pizzinato, A. (2003). Competência social infantil no contexto escolar: Alunos imigrantes em Porto Alegre. Dissertação de Mestrado não-publicada, Pontifícia Universidade Católica do Rio Grande do Sul, Porto Alegre, RS.

Pons, F., Harris, P. L., \& Rosnay, M. (2004) Emotion comprehension between 3 and 11 years: Developmental periods and hierarchical organization. European Journal of Developmental Psychology, 1, 127-151.

Repacholi, B., Slaughter, V., Pritchard, M., \& Gibbs, V. (2003). Theory of mind, machiavellianism, and social functioning in childhood. In B. Repacholi \& V. Slaughter (Eds.), Individual differences in theory of mind (pp. 67-98). New York: Psychology Press.

Ronald, A., Happé, F., Hughes, C., \& Plomin, R. (2005). Nice and nasty theory of mind in preschool children: Nature and nurture. Social Development, 14, 664-684.
Rose, A. J., \& Asher, S. R. (2000). Children's friendship. In C. Hendrick \& S. S. Hendrick (Eds.), Close relationships: A sourcebook (pp. 46-57). Thousand Oaks, CA: Sage.

Slaughter, V., Dennis, M. J., \& Pritchard, M. (2002). Theory of mind and peer acceptance in preschool children. British Journal of Developmental Psychology, 20, 545-564.

Storbeck, J., \& Clore, G. L. (2007). On the interdependence of cognition and emotion. Cognition and Emotion, 21, 12121237.

Sutton, J., Smith, P. K., \& Swettenham, J. (1999a). Bullying and 'theory of mind': A critique of the 'social skills deficit' view of anti-social behaviour. Social Development, 8, 117127.

Sutton, J., Smith, P. K., \& Swettenham, J. (1999b). Socially undesirable need not be incompetent: A response to Crick and Dodge. Social Development, 8, 132-134.

Watson, A. C., Nixon, C. L., Wilson, A., \& Capage, L. (1999). Social interaction skills and theory of mind in young children. Developmental Psychology, 35, 386-391. 